\title{
Physical and chemical properties of the AFGL 333 cloud
}

\author{
Takeshi Sakai ${ }^{1} \dagger$, Tomoharu Oka ${ }^{2}$ and Satoshi Yamamoto ${ }^{2}$ \\ ${ }^{1}$ Nobeyama, Radio Observatory, Japan \\ email: sakai@nro.nao.ac.jp \\ ${ }^{2}$ Department of Physics, Graduate School of Science, University of Tokyo, Japan
}

\begin{abstract}
We have found massive clumps without any sign of active star formation in the AFGL 333 cloud. We present a study of the physical and chemical properties of the AFGL 333 cloud.
\end{abstract}

The AFGL 333 cloud is located in the W 3 giant molecular cloud (W 3 GMC). It is known that the W 3 GMC involves three star forming clouds; W 3 Main, W $3(\mathrm{OH})$, and AFGL 333 (e.g. Thronson et al. 1985). The three clouds exhibit different star-forming activities in spite of their similarity in size and mass. The ratio of the infrared luminosities in W 3 Main, W 3(OH) and AFGL 333 is 1.0:0.25:0.07 (Thronson et al. 1980), suggesting that AFGL 333 is less active than W 3 Main and W $3(\mathrm{OH})$.

We have mapped the $\mathrm{C}^{0}{ }^{3} P_{1}{ }^{3} P_{0}([\mathrm{CI}])$ and $\mathrm{CO} J=3-2$ lines toward the $\mathrm{W} 3$ GMC by using the Mount Fuji Submillimeter Telescope (Sakai et al. 2006). The [CI] emission is found to be strong in the AFGL 333 cloud, where the ${ }^{12} \mathrm{CO} J=3-2$ emission is relatively weak. To investigate the origin of the strong [CI] emission in the AFGL 333 cloud, we have observed the AFGL 333 and W $3(\mathrm{OH})$ clouds in the CO isotopomer lines and the CCS and $\mathrm{N}_{2} \mathrm{H}^{+}$lines with the Nobeyama Radio Observatory $45 \mathrm{~m}$ telescope. We have found that $N\left(\mathrm{C}^{0}\right)$ linearly increases with $N(\mathrm{CO})$ up to $A_{V}$ of $50 \mathrm{mag}$. This indicates that $\mathrm{C}^{0}$ exists in the deep inside of the molecular clouds. The $\left[\mathrm{C}^{0}\right] /[\mathrm{CO}]$ and $[\mathrm{CCS}] /\left[\mathrm{N}_{2} \mathrm{H}^{+}\right]$ ratios tend to be higher in the AFGL 333 cloud than in the $\mathrm{W} 3(\mathrm{OH})$ cloud. These results may indicate the chemical youth of the AFGL 333 cloud relative to the $\mathrm{W} 3(\mathrm{OH})$ cloud.

In the AFGL 333 cloud, we have found two massive clumps (Clump A and B) without any sign of active star formation. They are highly gravitationally bound $\left(M_{\mathrm{VIR}} / M_{\mathrm{LTE}} \sim\right.$ 0.4 ), and the LTE mass is $2.3 \times 10^{3} M_{\odot}$ and $1.4 \times 10^{3} M_{\odot}$ for Clump A and Clump $\mathrm{B}$, respectively. These masses are comparable to those for on-going massive star-forming clumps. We have mapped the $\mathrm{CCS}, \mathrm{HC}_{3} \mathrm{~N}$ and $\mathrm{N}_{2} \mathrm{H}^{+}$emissions toward the clumps, and have found that the $\mathrm{CCS}$ and $\mathrm{HC}_{3} \mathrm{~N}$ emissions are stronger toward Clump $\mathrm{B}$ than toward Clump A. There are several YSO candidates (2MASS sources with $H-K>2$ ) in Clump A, while no YSO candidate is associated with Clump B. These results suggest that Clump $\mathrm{B}$ is younger than Clump A and is in a very early stage of cluster formation. Therefore Clump B is a very good target to understand the initial condition of cluster formation.

\section{References}

Sakai, T., Oka, T. \& Yamamoto, S. 2006, ApJ 649, 268

Thronson, H. A., Jr., Campbell, M. F. \& Hoffmann, W. F. I. 1980, ApJ 239, 533

Thronson, H. A., Jr., Lada, C. J. \& Hewagama, T. 1985, ApJ 297, 662

$\dagger$ Present address: Nobeyama, Minamimaki, Minamisaku, Nagano 384-1305, Japan 